\title{
Uncertain Decision-Making: A Mathematical Programming Perspective
}

\author{
Xiang Li, ${ }^{1}$ Dan Ralescu, ${ }^{2}$ Xiaofeng Xu, ${ }^{3}$ Lei Li, ${ }^{4}$ and Irene Diaz ${ }^{5}$ \\ ${ }^{1}$ School of Economics and Management, Beijing University of Chemical Technology, Beijing, China \\ ${ }^{2}$ Department of Mathematical Sciences, University of Cincinnati, Cincinnati, OH, USA \\ ${ }^{3}$ School of Economics and Management, China University of Petroleum, Qingdao, China \\ ${ }^{4}$ Faculty of Engineering, Hosei University, Tokyo, Japan \\ ${ }^{5}$ Department of Computer Science, University of Oviedo, Oviedo, Spain
}

Correspondence should be addressed to Xiang Li; lixiang@mail.buct.edu.cn

Received 20 March 2017; Accepted 21 March 2017; Published 23 April 2017

Copyright (C) 2017 Xiang Li et al. This is an open access article distributed under the Creative Commons Attribution License, which permits unrestricted use, distribution, and reproduction in any medium, provided the original work is properly cited.

Uncertain decision exists in various kinds of human behaviors, especially in coping with complex problems. There is no surprise that with the ever-increasing complexity of problems, uncertain decision will be made by different formal ways and technologies in dynamic environment, and it calls for innovative approaches to provide interpretable solutions and effective decisions. Mathematical programming with its broad ornamentation of technologies of linear programming, nonlinear programming, integer programming, uncertain programming, combinatorial optimization, and so on plays an important role in the formulating and solving uncertain problems.

This special issue aims to deliver a platform. On the platform, researchers coming from academe and industry can present the advanced methodologies to cope with uncertain decision-making and optimization problems. At the same time, they can also elaborate on the state-of-the-art case studies in selected areas of application through the usage of concepts of mathematical programming and reports on the linkages between methodology and practice of mathematical programming.

Once the call for papers was announced in February 2016, this special issue has attracted tremendous attention. In total, we received 46 manuscripts. After a rigorous review process, 18 papers have been finally accepted for publication.

To gain a better insight into the essence of the special issue, we offer brief highlights of the contributing papers.
Theory. The paper titled "Model and Simulation of Network Crisis Information Diffusion under Uncertain Environment" views the public as a series of cellular automata and sets up some cellular state evolution rules and then explores the diffusion rule of crisis information diffusion process and finds out the key factors of crisis information diffusion process and its influence on the diffusion scale and effect. A panel threshold model is used in "Noninterest Income and Performance of Commercial Banking in China" with balanced panel dataset of 16 listed Chinese commercial banks, for the period of 2007 to 2013, to investigate the relationship between noninterest income and performance. $\mathrm{X}$. N. Zhang et al. established an evaluation index system for the regional collaborative innovation system considering the uncertainty of collaborative systems. The collaborative uncertainty decision model is constructed to determine the regional innovation system's collaborative innovation effectiveness. The paper titled "Modified Bat Algorithm Based on Lévy Flight and Opposition Based Learning" proposes an improved BA called OBMLBA. In the proposed algorithm, a modified search equation with more useful information from the search experiences is introduced to generate a candidate solution. Furthermore, the concept of opposition based learning (OBL) is embedded to BA to enhance the diversity and convergence capability. The paper written by $\mathrm{H}$. Zhang et al. summarizes the factors that affect the city logistics distribution system. Starting from the research of factors that 
influence the reliability of city distribution system, further construction of city distribution system reliability influence model is built based on Bayesian networks. The complex problem is simplified by using the sub-Bayesian network, and an example is analyzed. The paper titled "Dependent-Chance Goal Programming for Water Resources Management under Uncertainty" introduces a new tool-uncertainty theory to deal with such uncertainty which is treated as uncertain variable with uncertainty distribution. And a dependentchance goal programming (DCGP) model is provided for water resources management under such circumstance. J. Song et al. analyze economy-energy-environment system's structure and components. G. Yang and H. Gao put forward an evaluation model of the uncertain risks of knowledge management. Based on set pair theory, a model is established to identify the knowledge management risk. Through the concept of connection number, knowledge management risk is divided into five levels.

Methodologies and Case Studies. X. Xu et al. in the paper entitled "Resource Allocation Optimization Model of Collaborative Logistics Network Based on Bilevel Programming" consider the uncertainty of time to establish a bilevel programming model with random constraints and propose a genetic simulated annealing hybrid intelligent algorithm to solve it. X. Shan et al. present a practical risk assessment method based on Bow-Tie model and Bayesian network for risk analysis of natural gas pipeline leakage. Q. Meng et al. propose a decision method for maximizing service quality in machinery industry under budget constraints, from the perspective of enterprise capacity and customer satisfaction. They formalize the relationship between customer satisfaction and sufficiency of service quality elements quantitatively and develop a novel nonlinear programming model to maximize service quality under budget constraints. $\mathrm{X}$. Gong and J. Lu investigate spillovers in the Capesize forward freight agreements (FFAs) markets before and after the global financial crisis. The results suggest that there are volatility spillover effects in certain Capesize FFAs routes, and the effects from spot rates to FFAs take place before crisis, yet they are bilateral after crisis. J. Liu et al. present an integrated method to assess the supply chain vulnerability. Combining the fuzzy theory and the gray theory, the correlation degree of each vulnerability indicator can be calculated and the target improvements can be carried out. In order to verify the effectiveness of the proposed method, they use Kendall's tau coefficient to measure the effect of different methods. S. C. Lu et al. build two competitive theory models. Through analyzing the equilibrium, one gets two main results: (1) according to different service area, the cities (Dalian and Tianjin) should have different price; (2) the two-sided market characters have an impact on their strategies. The paper titled "Optimal Uncertainty Decision of Innovation Products Output Based on Return Collection" studies the optimal single-period production decision between reused parts and conventional new parts in complementary and substitute relationships. It further gives the analytical expressions for satisfying the optimal production and provides some numerical examples. A bilevel fuzzy chance constrained model is developed in "Bilevel Fuzzy Chance Constrained Hospital Outpatient Appointment Scheduling Model" to solve the hospital outpatient appointment scheduling problem based on revenue management. Then use chance operator to handle the model with fuzzy parameters and equivalently transform the appointment scheduling model into a crisp model. Moreover, interactive algorithm based on satisfaction is employed to convert the bilevel programming into a single level programming, in order to make it solvable. Li et al. developed a novel multiobjective optimization model with mixed uncertain coefficients, which maximizes the total profits and minimizes the percentage of items delivered late, percentage of items rejected, and total loss cost due to supplier dysfunction.

We envision that the papers published in this special issue would be of interest to researchers and practitioners and help identify further research directions. We also hope that the readers can find the material of this special issue both interesting and inspiring when exploring the field of optimization under uncertainty.

\section{Acknowledgments}

As Guest Editors, we would also like to thank all the referees for their support and professional service and express the gratitude to all authors for their submissions to this special issue. Without the support of the authors and the referees, it would have been impossible to make this special a reality.

Xiang Li
Dan Ralescu
Xiaofeng Xu
Lei Li
Irene Diaz



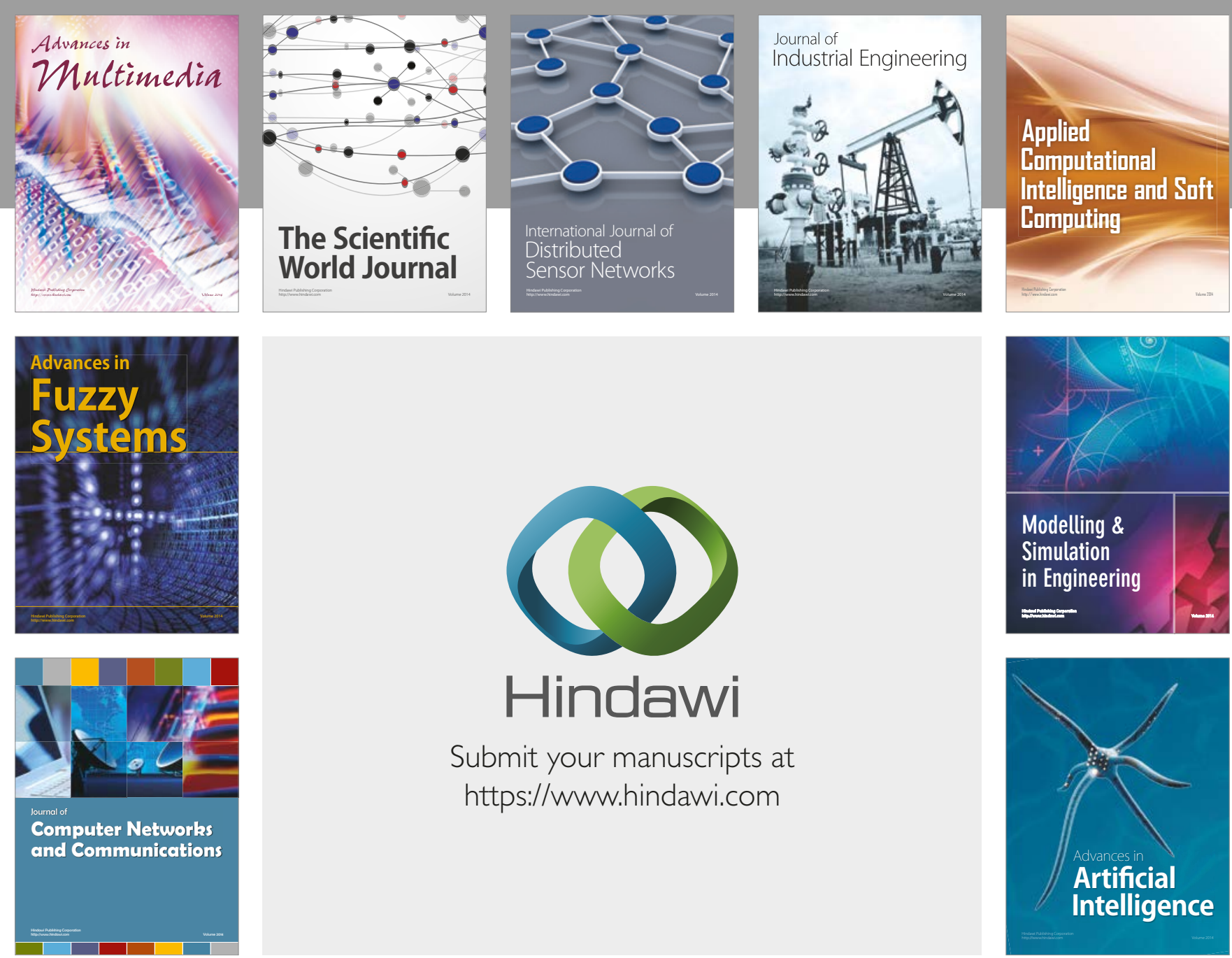

\section{Hindawi}

Submit your manuscripts at

https://www.hindawi.com
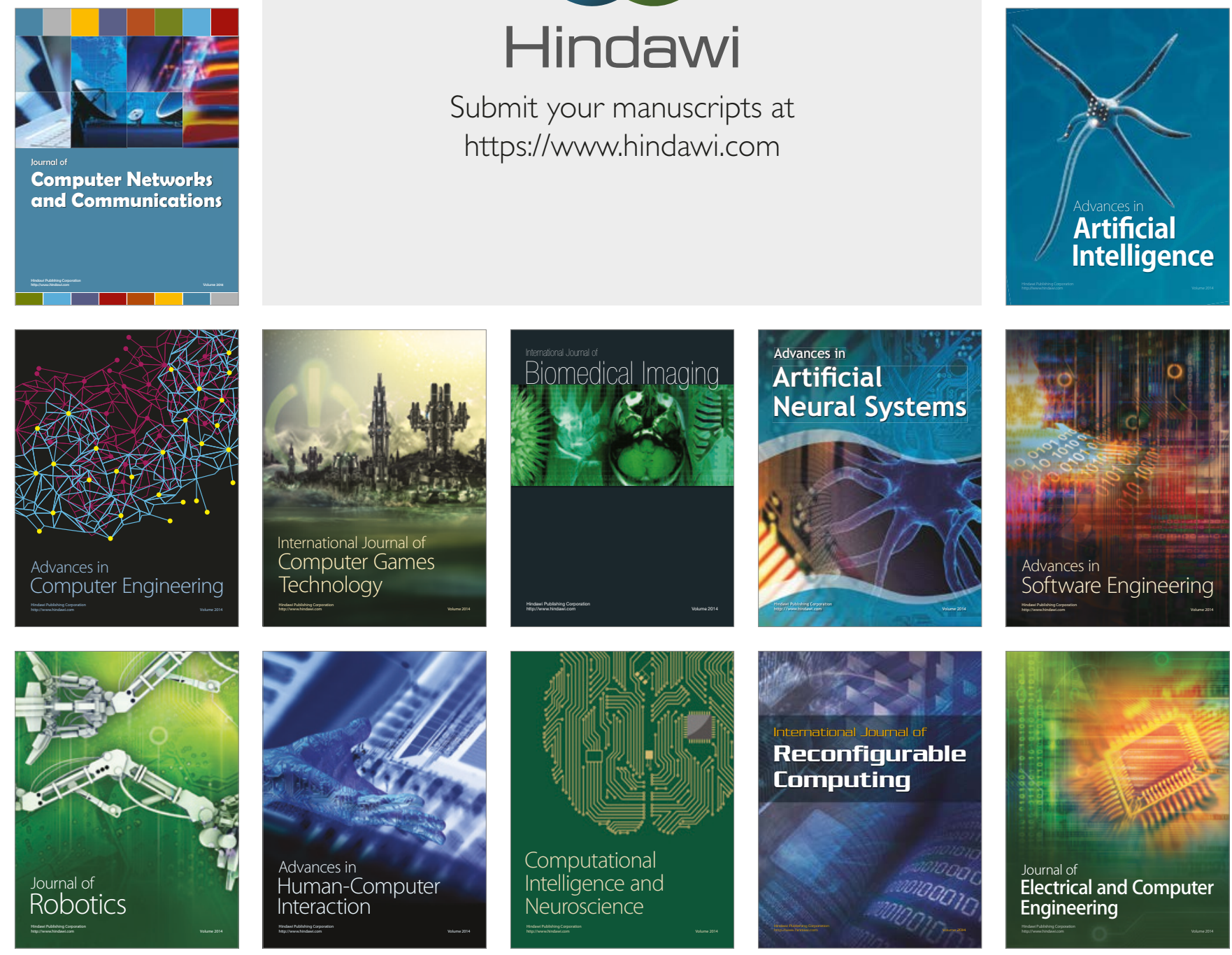